\title{
Laboratory animal allergy is preventable in modern research facilities
}

\author{
Johanna R. Feary ${ }^{1,2}$, Susan J. Schofield ${ }^{2}$, Jennifer Canizales ${ }^{2}$, \\ Bernadette Fitzgerald ${ }^{1}$, James Potts ${ }^{2}$, Meinir Jones ${ }^{2}$ and Paul Cullinan ${ }^{1,2}$
}

Affiliations: 'Dept of Occupational Lung Disease, Royal Brompton and Harefield NHS Foundation Trust, London, UK. ${ }^{2}$ Occupational and Environmental Medicine, National Heart and Lung Institute, Imperial College London, London, UK.

Correspondence: Johanna R. Feary, National Heart and Lung Institute, Imperial College London, Emmanuel Kaye Building, 1b Manresa Road, London, SW3 6LR, UK. E-mail: j.feary@imperial.ac.uk

@ERSpublications

Laboratory animal allergy, an important form of occupational asthma, can be prevented in modern research units using a multifaceted approach including individually ventilated cages to contain aeroallergen exposure and careful use of respiratory protection http://ow.ly/r6dM30oj3Ij

Cite this article as: Feary JR, Schofield SJ, Canizales J, et al. Laboratory animal allergy is preventable in modern research facilities. Eur Respir J 2019; 53: 1900171 [https://doi.org/10.1183/13993003.00171-2019].

\section{ABSTRACT}

Background: Historical data suggest 15\% of laboratory animal workers develop IgE sensitisation and $10 \%$ symptoms of laboratory animal allergy (LAA), including occupational asthma. Individually ventilated cages (IVCs) are replacing conventional open cages; we sought to evaluate their impact on the development of LAA.

Methods: We surveyed 750 laboratory animal workers and measured airborne Mus m 1 (mouse allergen) levels in seven UK institutions. We compared the prevalence of sensitisation to mouse proteins (by specific IgE assay or skin prick test) and of work-related allergic symptoms in IVC-only and open cage units.

Results: Full-shift Mus $\mathrm{m} 1$ levels were lower in IVC than open cage units (geometric mean 1.00 (95\% CI $0.73-1.36$ ) versus 8.35 (95\% CI 6.97-9.95) $\mathrm{ng} \cdot \mathrm{m}^{-3} ; \mathrm{p}<0.001$ ), but varied eight-fold across the IVC units (geometric mean range $0.33-4.12 \mathrm{ng} \cdot \mathrm{m}^{-3}$ ). Primary analyses on data from 216 participants with $\leqslant 3$ years exposure to mice revealed a lower prevalence of sensitisation in those working in IVC units compared with conventional cage units $(2.4 \%(\mathrm{n}=2)$ versus $9.8 \%(\mathrm{n}=13) ; \mathrm{p}=0.052)$. Sensitisation in IVC units varied from $0 \%$ to $12.5 \%$; the use of fitted respiratory protection was less common in IVC units where prevalence of sensitisation was higher. Work-related allergy symptoms were more frequently reported by mousesensitised individuals $(46.7 \%$ versus $10.9 \%$; $\mathrm{p}<0.001)$ and only by those working in open cage units.

Conclusion: In contemporary practice, LAA is now largely preventable with the use of IVC systems and the judicious use of appropriate respiratory protection. 


\section{Introduction}

Laboratory animal allergy (LAA) is the manifestation of an IgE-associated sensitisation to animal proteins, and is a common cause of occupational allergy and asthma. Evidence of an exposure-response relationship between airborne rat urinary proteins and LAA has long been established, with increasing risks of $\operatorname{IgE}$ sensitisation and LAA at higher levels of exposure [1-3]; the evidence for allergy to mouse proteins is less clear $[4,5]$.

Previous studies of LAA, largely of employees in facilities using open cages to house rats, suggest $\sim 15 \%$ of exposed workers developed specific IgE sensitisation and $10 \%$ clinically apparent disease [6]. Recent, significant changes in animal research institute practices have rendered much of the published literature on LAA potentially outdated. Importantly, conventional open cages are being replaced by expensive individually ventilated cages (IVCs) to maintain gnotobiotic colonies in a microbiologically controlled environment; IVCs are generally used in conjunction with other engineering control "systems" such as use of a hood with local exhaust ventilation when handing mice and may also have the effect of reducing, in some circumstances, levels of aeroallergen $[5,7,8]$. In addition, with the advance of genetic manipulation, mice, rather than rats, are now the major animal model, e.g. in 2017 mice were used in $74 \%$ of the 3.79 million procedures carried out on animals in Great Britain [9]. The major mouse urinary protein, Mus $\mathrm{m} \mathrm{1,}$ is well characterised, excreted mainly in urine [10] and rendered airborne by tasks that involve handling of mice or their bedding material.

To date, there has been no published intervention study to evaluate the effectiveness of reducing levels of aeroallergens in animal facilities on decreasing the frequency of sensitisation and allergic disease. We exploited the introduction of IVCs to some, but not all, animal units to investigate whether aeroallergen levels are lower in IVC facilities and whether any reduction in aeroallergen levels is associated with a lower incidence of sensitisation to mouse proteins and LAA. As a secondary aim, we investigated if any individual factors might confound the relationship between aeroallergen exposure and specific $\operatorname{IgE}$ sensitisation.

\section{Methods}

Between July 2014 and March 2017 we surveyed employees of seven UK research institutions housing mice (with or without rats). We identified potential participants through lists of registered animal users and research group members. All employees with a minimum of 4 months of (current) occupational exposure to mice were eligible. Ethical permission was granted by the Office for Research Ethics Committees Northern Ireland (13/NI/0208).

The survey was conducted on-site. After taking informed consent we carried out skin prick tests to extracts of common aeroallergens (cat, grass and Dermatophagoides pteronyssinus), mouse and rat epithelia, and histamine (positive) and saline (negative) controls (Diagenics, Milton Keynes, UK and Stallergenes, London UK). Blood samples were taken for assay of serum specific IgE to mouse urine and epithelium (Thermo Fisher Scientific, Uppsala, Sweden). We invited participants to complete an online questionnaire that included questions about current and previous exposure to laboratory animals, use of personal protective equipment, symptoms and prior diagnoses of work-related allergy, and pertinent previous health conditions.

We determined sensitisation by a positive specific IgE to mouse urine (e72) or mouse epithelium (e71) (ImmunoCAP; Phadia, Uppsala, Sweden; $\geqslant 0.35 \mathrm{kU} \cdot \mathrm{L}^{-1}$ ), a positive saline-adjusted skin prick test (wheal diameter $\geqslant 2 \mathrm{~mm}$ ) to mouse epithelium, or both $[11,12]$. We defined atopy by a positive saline-adjusted skin prick test to one or more common aeroallergens.

In addition, we measured background levels of, and personal exposure to, Mus $\mathrm{m} 1$ in the different units; the methods are reported in the supplementary material.

\section{Statistical methods}

Our primary aim was to compare the prevalence of sensitisation between those who had only ever worked in IVC units with those with previous or current work in a conventional open cage unit. To minimise the impact of survivor biases (the "healthy worker effect") we restricted our primary analyses to workers with $\leqslant 3$ years of occupational exposure to mice $[1,13]$.

The distributions of aeroallergen measurements were log-normal and are summarised by their geometric means and $95 \%$ confidence intervals. We present other continuous variables by their mean and standard deviation or median and range, and categorical variables as percentages. For comparisons between groups we used the Kruskal-Wallis test for nonparametric continuous data and the Chi-squared test for proportions (or Fisher's exact test where appropriate). All tests were two sided. We undertook logistic regression to test the independent associations between sensitisation and work with IVC/open cages, 
smoking and atopy. Finally, we conducted an ecological analysis of the relationship between sensitisation and unit. We used Stata version 14 (StataCorp, College Station, TX, USA) for all analyses.

\section{Results}

Aeroallergen levels are consistently lower in IVC units

Background Mus $\mathrm{m} 1$ levels were significantly lower in IVC units than open cage units ( $\mathrm{n}=189$ samples; geometric mean 0.04 (95\% CI $0.02-0.10$ ) versus 0.53 (95\% CI $0.30-0.95)$ ng.m $\left.{ }^{-3} ; \mathrm{p}<0.001\right)$. Similarly, we collected 241 full-shift, representative, personal exposure samples (median (range) sampling time of 365 $(180-550) \mathrm{min}$ ), and found that Mus $\mathrm{m} 1$ levels across shifts were significantly and consistently higher in open cage and mixed IVC/open cage units than in IVC units (geometric mean 8.35 (95\% CI 6.97-9.95) versus $1.00(95 \%$ CI $\left.0.73-1.36) \mathrm{ng} \cdot \mathrm{m}^{-3} ; \mathrm{p}<0.001\right)$. However, there was an eight-fold difference in mean personal aeroallergen exposures across the IVC units (geometric mean range $0.33-4.12 \mathrm{ng} \cdot \mathrm{m}^{-3}$ ).

Prevalence of sensitisation to mouse proteins is lower in participants working in IVC units

We recruited 750 participants (response rate 85\%) and complete information on 743 individuals. Of the seven surveyed institutions, five housed mice only in IVCs (units A, C, D, E and G) and one (unit B) had mixed housing where employees worked with mice predominantly in open cages but also a few IVCs; the final institution (unit F) was categorised into two as employees either worked in open cage (Fo) or IVC (FI) units. Two-thirds (65.8\%) of all participants were research scientists; overall, $10.4 \%(\mathrm{n}=77)$ were sensitised to mice (table 1).

We restricted further analyses to the 216 (29.1\%) participants with $\leqslant 3$ years exposure to mice. This population was younger and more frequently atopic $(44.1 \%)$ than the total population (38.9\%), and a higher proportion of participants had only ever worked in IVC units (38.4\% versus 28.1\%) (table 1 ). $15(6.9 \%)$ participants in this population were sensitised to mouse proteins; of these, 12 had had no previous occupational or pet exposure to mice, suggesting that sensitisation had occurred in their current job.

TABLE 1 Characteristics of study participants: total population and restricted population $1 \leqslant 3$ years work with mice)

Total population

Subjects
Male
Age years
Current smoker
Atopic $^{\#}$
Sensitised to mice
Sensitised to rats
Sensitised to rats and mice
No occupational exposure to mice prior to current post
Years exposed to mice
Job title
Technician
Scientist
Other
Unit
A
B
C
D
E
Fo
FI
G
Cage type
IVC only ever
Open/mixed ever

743
$312(42.0)$
$31.7(17.8-67.2)$
$85(11.4)$
$286(38.9)$
$77(10.4)$
$32(4.4)$
$29(10.8)$
$345(46.5)$
$6.0(0.5-42.0)$

206 (27.7)

$489(65.8)$

$48(6.5)$

$60(8.1)$

$178(24.0)$

$82(11.0)$

88 (11.8)

77 (10.4)

$161(21.6)$

$76(10.2)$

21 (2.8)

209 (28.1)

534 (71.9)
Restricted population

216

86 (39.8)

$25.8(17.8-61.3)$

31 (14.4)

94 (44.1)

$15(6.9)$

7 (3.3)

$6(2.8)$

$187(86.6)$

$1.8(0.5-3.0)$

63 (29.2)

148 (68.5)

5 (2.3)

$13(6.0)$

$60(27.8)$

$19(8.8)$

$15(6.9)$

23 (10.7)

$64(29.6)$

$21(9.7)$

$1(0.5)$

83 (38.4)

133 (61.6)

Data are presented as $\mathrm{n}, \mathrm{n}(\%)$ or median (range). " : saline-adjusted wheal of $\geqslant 2 \mathrm{~mm}$ to grass, Dermatophagoides pteronyssinus or cat on skin prick testing; " : saline-adjusted wheal of $\geqslant 2 \mathrm{~mm}$ to mouse or positive serum specific lgE to mouse epithelium or mouse urine: ${ }^{+}$: included microbiologists, managers, veterinarians and domestic assistants. 
83 participants had only worked in IVC units. Of these, two (2.4\%) were sensitised, a proportion lower than in those who had worked in open cage units $(9.8 \% ; \mathrm{p}=0.052)$ (table 2).

Individual factors associated with sensitisation to mouse proteins

In participants sensitised to mice, the prevalence of atopy was significantly higher $(80.0 \%$ versus $40.8 \%$; $\mathrm{p}=0.005)$ as was current smoking $(46.7 \%$ versus $11.9 \% ; \mathrm{p}<0.001)$. Age, job title and history of pet rodent ownership were not significantly associated with sensitisation, nor were having had a mask fit test or the reported wearing of a face mask while carrying out tasks likely to be associated with high allergen exposure (handling mice or their soiled bedding) (table 2). Both of the sensitised individuals who had only ever worked in IVC units were scientists; of the 13 participants sensitised to mice and who had worked in open cage units, eight (61.5\%) were scientists, four $(30.8 \%)$ were technicians and one $(7.7 \%)$ was a unit manager who had previously been an animal technician.

Logistic regression analysis showed that both the crude and adjusted odds of sensitisation were lower in those who had only ever worked in IVC units, although the difference was not statistically significant (adjusted OR 0.24 (95\% CI 0.05-1.15); p=0.074) (table 3). The odds of sensitisation to mice were significantly increased in current smokers (adjusted OR 6.43 (95\% CI 1.96-21.10); $\mathrm{p}=0.002$ ) and in those who were atopic to common aeroallergens (adjusted OR 6.93 (95\% CI 1.77-27.14); p=0.005).

Wearing of fitted respiratory protection associated with sensitisation at an ecological level We noted that the prevalence of sensitisation to mouse proteins varied from $6.7 \%$ to $10.4 \%$ in the open cage units (12 cases) and from $0 \%$ to $12.5 \%$ in the IVC units (three cases). Figure 1 presents an ecological analysis across different units. Aeroallergen levels were consistently higher in the open cage units compared with the IVC units; however, neither cage type nor allergen level fully explained the variation in prevalence of sensitisation between the units. The prevalence of smoking (4.4-19.5\%) and of atopy (33.3$57.1 \%$ ) varied between the units but, at an ecological level, there was no obvious pattern relating these variables to mouse sensitisation (data not shown). However, there were higher reported rates of mask fit tests and use of masks at the three units with zero or low rates of sensitisation.

\section{Symptoms and identification of LAA}

In the population with $\leqslant 3$ years exposure to mice, work-related allergy symptoms were reported more often by those sensitised to mouse proteins than those who were not sensitised (46.7\% versus $10.9 \%$; $\mathrm{p}<0.001$ ); these differences were significant for work-related nasal and ocular symptoms (table 4). Seven

\begin{tabular}{|c|c|c|c|}
\hline & Mouse sensitised " & Not sensitised & p-value \\
\hline Subjects & $15(6.9)$ & $201(93.1)$ & \\
\hline Male & 5 (33.3) & 81 (40.3) & 0.595 \\
\hline Age years & $25.3(21.8-42.9)$ & $25.8(17.8-61.3)$ & 0.841 \\
\hline Current smoker & $7(46.7)$ & $24(11.9)$ & $<0.001$ \\
\hline Atopic ${ }^{\#}$ & $12(80.0)$ & $82(40.8)$ & 0.005 \\
\hline Job title & & & 0.472 \\
\hline Technician & $4(26.7)$ & $59(29.4)$ & \\
\hline Scientist & $10(66.7)$ & 138 (68.7) & \\
\hline Other & $1(6.7)$ & $4(2.0)$ & \\
\hline Cage type & & & 0.052 \\
\hline IVC only ever & 2 (13.3) & 81 (40.3) & \\
\hline Open/mixed ever & $13(86.7)$ & $120(59.7)$ & \\
\hline Pet mice or rats ever & $3(20.0)$ & $23(11.4)$ & 0.400 \\
\hline Rat sensitised $^{+}$ & $6(40.0)$ & $1(0.5)$ & $<0.001$ \\
\hline Mask fit test & $6(40.0)$ & 87 (43.3) & 0.800 \\
\hline $\begin{array}{l}\text { Wearing of mask for tasks likely to be } \\
\text { associated with high allergen exposure }\end{array}$ & 5 (33.3) & $72(36.4)$ & 0.814 \\
\hline
\end{tabular}

Data are presented as $\mathrm{n}(\%)$ or median (range), unless otherwise stated. \#: saline-adjusted wheal of $\geqslant 2 \mathrm{~mm}$ to grass, Dermatophagoides pteronyssinus or cat on skin prick testing; ": saline-adjusted wheal of $\geqslant 2 \mathrm{~mm}$ to mouse or positive specific IgE to mouse epithelium or mouse urine; ${ }^{+}$: saline-adjusted wheal of $\geqslant 2 \mathrm{~mm}$ to rat epithelium on skin prick testing. $p<0.05$ considered significant. 
TABLE 3 Unadjusted and adjusted logistic regression of the association between sensitisation and individually ventilated cages (IVCs)/open cages in those with $\leqslant 3$ years work with mice

\begin{tabular}{lccc} 
& Unadjusted OR (95\% CI) & Adjusted OR (95\%) & p-value \\
\hline IVC only ever & $0.23(0.05-1.04)$ & $0.24(0.05-1.15)$ & 0.074 \\
Current smoking & $6.45(2.15-19.39)$ & $6.43(1.96-21.10)$ & 0.002 \\
Atopic $^{\text {T }}$ & $5.66(1.55-20.69)$ & $6.93(1.77-27.14)$ & 0.005 \\
\hline
\end{tabular}

\#: adjusted for current smoking and atopy; ": saline-adjusted wheal of $\geqslant 2 \mathrm{~mm}$ to grass, Dermatophagoides pteronyssinus or cat on skin prick testing. $p<0.05$ considered significant.

individuals were sensitised and reported work-related nasal or respiratory symptoms; all had worked in an open or mixed cage unit.

Only a minority of participants with symptomatic sensitisation to mouse proteins had been formally diagnosed with LAA as part of a health surveillance programme (two out of seven (28.6\%) in the restricted population and 17 out of $35(48.6 \%)$ in the total population). There were three individuals in the restricted population and seven in the total population who had previously been told they were allergic to mice by a healthcare professional but on testing had no immunological evidence of sensitisation to mouse proteins.

\section{Discussion}

Our findings, from a large cross-sectional multicentre study of laboratory animal workers, suggest it is now possible to run a modern mouse research unit with a very low risk of sensitising individuals, indicating that LAA is largely, if not wholly, preventable. The prevalence of sensitisation to mice in our study was lower than recently reported $[5,14]$, particularly so in the IVC units, with three of the seven such units not having any recently employed workers who were sensitised. The low prevalence in sensitisation reflects, as we have demonstrated, better aeroallergen control with lower ambient levels of Mus $\mathrm{m} 1$ with the use of IVCs. However, the considerable variation in sensitisation between different IVC units, despite similar background aeroallergen levels, suggests this is not the sole explanation and we observed differences in practice between the IVC units that may be relevant. In our ecological analysis we demonstrated variation in the proportion of employees who reported having a mask fit test and wearing a mask during tasks likely to be associated with high allergen exposure. Units where the prevalence of sensitisation was very low or zero had higher proportions of staff who reported the regular use of fitted

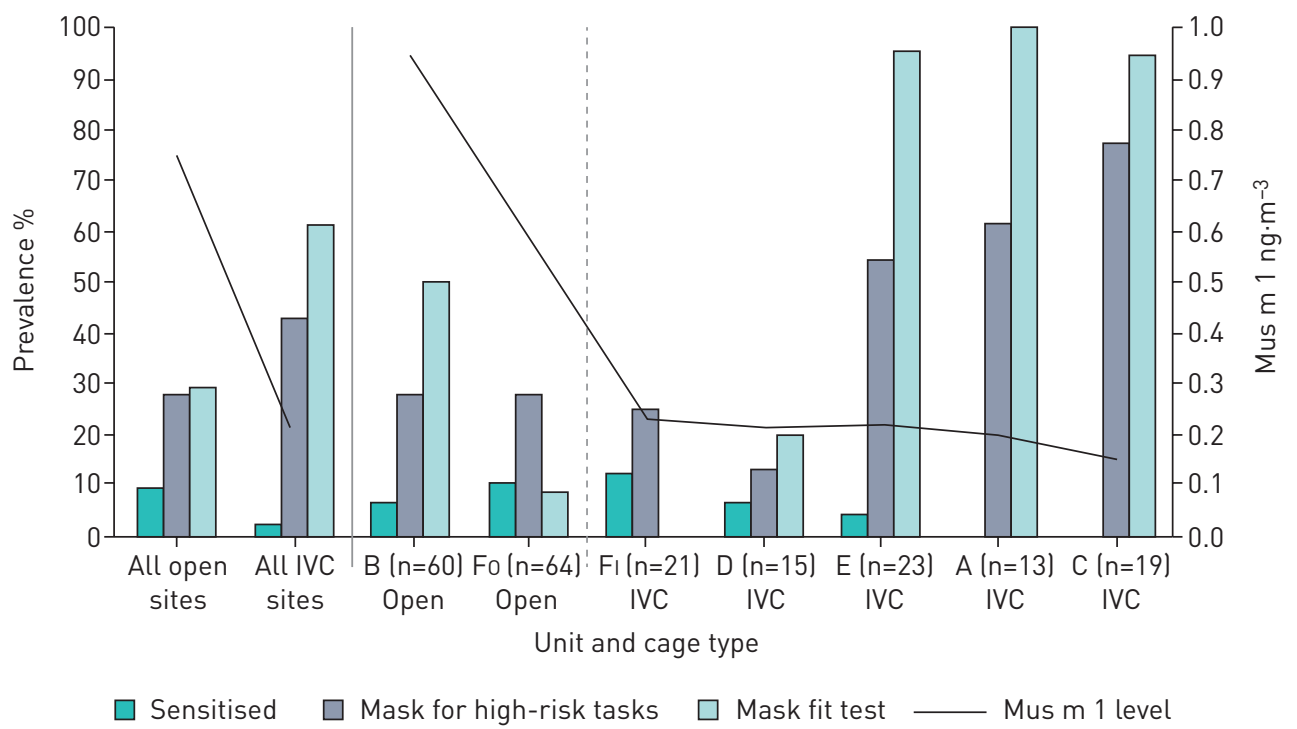

FIGURE 1 Prevalence of sensitisation to mice by unit ${ }^{\#}$, mask wear for tasks likely to be associated with high allergen exposure (labelled "high risk") and mask fit tests, and background levels of aeroallergen to Mus m 1 in those with $\leqslant 3$ years exposure to mice. IVC: individually ventilated cage. \#: see main text for description of units lat unit $G$ only a single individual had $\leqslant 3$ years exposure to mice; this unit is therefore not included in the figure). 
TABLE 4 Work-related allergy symptoms and other allergic diseases by sensitisation to mouse proteins in those with $\leqslant 3$ years work with mice

\begin{tabular}{lccc} 
& Mouse sensitised $^{\#}$ & Not sensitised $^{\text {p-value }}$ \\
\hline Subjects & $5(6.9)$ & $201(93.1)$ & \\
Work-related respiratory symptoms & $2(13.3)$ & $4(2.0)$ & 0.058 \\
Self-reported current asthma (last 12 months) & $1(6.7)$ & $18(9.0)$ & 1.000 \\
Work-related nasal symptoms & $7(46.7)$ & $16(8.0)$ & $<0.001$ \\
Self-reported current hay fever (last 12 months) & $9(60.0)$ & $66(32.8)$ & 0.033 \\
Work-related ocular symptoms & $5(33.3)$ & $10(5.0)$ & $<0.001$ \\
$\geqslant 1$ work-related symptom (respiratory, nasal or ocular) & $7(46.7)$ & $22(10.9)$ & $<0.001$ \\
\hline
\end{tabular}

Data are presented as $\mathrm{n}(\%)$, unless otherwise stated. ${ }^{\#}$ : saline-adjusted wheal of $\geqslant 2 \mathrm{~mm}$ to mouse or positive serum specific $\lg E$ to mouse epithelium or mouse urine. $p<0.05$ considered significant.

respiratory protection: this practice may be a surrogate for careful attention to UK guidance on respiratory protection in animal units [15]. Importantly, work-related allergy symptoms in individuals sensitised to mice were uniquely reported in those working in open or mixed cage units, suggesting that higher aeroallergen levels may be required to provoke symptoms following sensitisation.

In keeping with other studies $[1,14]$ and with some other causes of occupational asthma [16] we found atopy was strongly associated with sensitisation to mice. We also found a strong association between current smoking and sensitisation; this relationship is less consistent in the literature. VenABLEs et al. [12] found a significant increase in risk of sensitisation to rats in smokers; a second study also showed that sensitisation to rats was more common in smokers, but this association was lost in a multivariable analysis [17]. However, most studies have found no association between smoking and sensitisation to, primarily, rats but also other laboratory animal allergens [1, 18-20]. We suggest that once environmental exposures have been tightly controlled, factors that determine individual susceptibility become more prominent. A similar observation was made in precious metal refinery workers where the influence of human leukocyte antigen phenotype was more pronounced at lower levels of platinum salt exposure [21].

Most reports of laboratory animal workers relate to work with rats and were published in the (rapidly disappearing) era of open-caged housing. There are relatively few reports of sensitisation to mice in laboratory workers; all relate to single centres using conventional cages, to smaller populations than in our study and, with one exception, were published $>20$ years ago. An Australian study of 121 workers with a median exposure history of 3 years (range 3 months to 41 years) found a prevalence of $17.4 \%$ based on a skin prick test result of $\geqslant 2 \mathrm{~mm}$ to mouse allergens [22]. A Dutch study reported a 2-year incidence of $16 \%$ in sensitisation (positive skin prick test result of $\geqslant 3 \mathrm{~mm}$ to mouse proteins or positive specific IgE to mice) in recently exposed laboratory animal workers [4]. In a study from Italy, two individuals from 75 workers who were followed for a mean $\pm \mathrm{SD}$ of $3.84 \pm 1.5$ years developed sensitisation (positive skin prick test of $\geqslant 3 \mathrm{~mm}$ to mouse extracts), but it is unclear if all these workers were exposed to mice [23]. A longitudinal study of 179 employees in a mouse breeding unit in the USA reported a 2-year incidence of $23 \%$ [5]. Possible explanations for this strikingly high incidence are the higher proportions of ever-smokers and participants who were atopic compared with our study ( $45 \%$ and $51 \%$, respectively) or that breeding units differ from other research units, e.g. in procedures carried out and in stocking density of mice. However, a recent study from Sweden of 70 animal workers (of whom 14\% were smokers and $37 \%$ were atopic) reported a similar 2-year incidence of 19\%; in that study, animals were housed in both IVCs and open cages, but no data were reported on differences in sensitisation by cage use [14].

Generally, data relating to the number of exposed laboratory animal workers and the various controls in place are not well recorded; globally the number is estimated to be several hundred thousand [24]. In the UK around 12000 people work with mice at 160 different establishments, primarily academic and medical research institutions $[9,25]$. In the USA, there was a low response rate $(19.2 \%)$ to an online survey of 1033 institutions identified by the National Institutes of Health Office of Laboratory Animal Welfare [26]. Of the 198 institutions who did respond, the majority (84\%) were academic or research organisations and thus have similar populations to those we studied. The authors reported considerable variability in workplace practices to reduce the risk of LAA, e.g. IVCs were used by $65 \%$ of institutions and fewer than one-third had compulsory wearing of respiratory protection.

The risks of LAA are highest in the early years of employment $[1,13,16]$ and an estimated $95 \%$ of individuals who develop LAA do so in the first 3 years of exposure [13]. By restricting our main analyses to those employed within this window we aimed to capture most incident cases and to mitigate the impact 
of any "healthy worker effect" [1], a well-recognised limitation of cross-sectional occupational surveys. Moreover, we were primarily interested in the risks of contemporary laboratory animal work. In the restricted population, $38 \%$ had only ever worked with IVCs compared with $28 \%$ of the total population, reflecting the increasing use of IVCs in research units. The higher prevalence of atopy in the restricted population (44.1\% versus $38.9 \%$ ) may reflect a birth cohort effect or the differential movement of atopic individuals away from animal work due to development of LAA in previous years.

Over half $(53.3 \%)$ of those sensitised to mice reported no work-related nasal, ocular or respiratory symptoms. Asymptomatic sensitisation is well recognised and may reflect a permanent state or a transition phase from immunological sensitisation to symptoms. As discussed earlier, symptoms may only manifest in environments where allergen levels are higher. Despite the confidential nature of our survey, individuals may have been reluctant to admit or report symptoms. In the total study population there were 46 sensitised individuals with work-related symptoms who appeared not to have been told they were allergic to mice, suggesting LAA is underdetected, leaving individuals without advice on how best to manage their exposures (both in the short and longer term) and vulnerable to developing persistent asthma. Moreover, seven individuals who were not sensitised to mice had been told they had LAA, suggesting an alternative explanation for their symptoms.

A strength of our survey is that it was multicentred and included both "state-of-the-art" animal facilities and less modernised units. Our findings reflect a wide range of practices and should be broadly generalisable. We acknowledge that some employees who developed LAA very early in their career may have left their workplace and that this could account for the low rates of sensitisation observed at some units. We were unable to obtain comprehensive lists of those who had left employment during our recruitment period, but review of referrals of suspected cases of LAA to our clinical service (standard practice for all the surveyed institutions) for the 5 years prior to recruitment found only three cases of confirmed LAA (all from open cage units) that had resulted in the individuals ceasing animal exposure. This suggests the low sensitisation rate is unlikely to be due to a survival effect; indeed, if there was a survival effect we had not captured, then the difference between the IVC and open cage groups would be even greater. It may be that new employees have not had sufficient exposure to develop sensitisation, leading to an underestimation of cases of LAA, but the proportion of participants with $<1$ and 1-2 years of exposure to mice was similar between units (data not shown). Our high response rate helped to minimise the risk of participator bias. Our study may have been carried out in units where management proactively protect the health of their workers, resulting in an underestimate of the general prevalence of sensitisation, although the wide disparities in mask fit testing suggest this was not universally true. The prevalence of mouse sensitisation was lower than anticipated and the number of cases in our restricted population was small, which limited our ability to test differences between units. Finally, this is a cross-sectional study and thus it is not possible to attribute with any certainty causal relationships to observed associations. Due to the high costs of IVCs, a randomised controlled trial of introduction of IVCs into animal units is not practical, and the low prevalence of sensitisation means that a longitudinal study of the incidence of LAA in open cage and IVC units would require a very large number of participants to be adequately powered.

The use of animal works will, for the foreseeable future, remain a crucial part of research in respiratory and other medicine [27]. LAA is a condition that historically afflicted up to $20 \%$ of a highly trained workforce with important health and economic consequences for the affected individual, their employing institutions and the wider scientific research community. Our findings indicate that LAA may be very largely preventable in modern animal research units by using a multifaceted approach, although at lower levels of aeroallergen exposure individual factors may remain relevant to its development.

Acknowledgements: We gratefully acknowledge the assistance of the SPIRAL (Safe Practice In Reducing Allergy in Laboratories) study Steering Committee, the management and occupational health departments at each study site, and all the study participants.

Conflict of interest: None declared.

Support statement: This work was funded by the National Institute for Health Research. Funding information for this article has been deposited with the Crossref Funder Registry.

\section{References}

1 Cullinan P, Cook A, Gordon S, et al. Allergen exposure, atopy and smoking as determinants of allergy to rats in a cohort of laboratory employees. Eur Respir J 1999; 13: 1139-1143.

2 Jeal H, Draper A, Harris J, et al. Modified Th2 responses at high-dose exposures to allergen: using an occupational model. Am J Respir Crit Care Med 2006; 174: 21-25.

3 Nieuwenhuijsen MJ, Putcha V, Gordon S, et al. Exposure-response relations among laboratory animal workers exposed to rats. Occup Environ Med 2003; 60: 104-108. 
4 Krop EJ, Doekes G, Heederik DJ, et al. IgG4 antibodies against rodents in laboratory animal workers do not protect against allergic sensitization. Allergy 2011; 66: 517-522.

5 Peng RD, Paigen B, Eggleston PA, et al. Both the variability and level of mouse allergen exposure influence the phenotype of the immune response in workers at a mouse facility. J Allergy Clin Immunol 2011; 128: 390-396.

6 Jeal H, Jones M, Cullinan P. Epidemiology of laboratory animal allergy. In: Sigsgaard T, Heederik D, eds. Occupational Asthma (Progress in Inflammation Research). Basel, Birkhauser, 2010; pp. 33-35.

7 Gordon S, Fisher SW, Raymond RH. Elimination of mouse allergens in the working environment: assessment of individually ventilated cage systems and ventilated cabinets in the containment of mouse allergens. J Allergy Clin Immunol 2001; 108: 288-294.

8 Renstrom A, Bjoring G, Hoglund AU. Evaluation of individually ventilated cage systems for laboratory rodents: occupational health aspects. Lab Anim 2001; 35: 42-50.

9 Home Office. Annual Statistics of Scientific Procedures on Living Animals, Great Britain. 2017. www.gov.uk/ government/uploads/system/uploads/attachment_data/file/627284/

annual-statistics-scientific-procedures-living-animals-2016.pdf Date last accessed: February 21, 2019.

10 Lorusso JR, Moffat S, Ohman JL Jr. Immunologic and biochemical properties of the major mouse urinary allergen (Mus m 1). J Allergy Clin Immunol 1986; 78: 928-937.

11 Johannsen H, Nolan R, Pascoe EM, et al. Skin prick testing and peanut-specific IgE can predict peanut challenge outcomes in preschoolchildren with peanut sensitization. Clin Exp Allergy 2011; 41: 994-1000.

12 Venables KM, Upton JL, Hawkins ER, et al. Smoking, atopy, and laboratory animal allergy. Br J Ind Med 1988; 45: 667-671.

13 Gross NJ. Allergy to laboratory animals: epidemiologic, clinical, and physiologic aspects, and a trial of cromolyn in its management. J Allergy Clin Immunol 1980; 66: 158-165.

14 Palmberg L, Sundblad BM, Lindberg A, et al. Long term effect and allergic sensitization in newly employed workers in laboratory animal facilities. Respir Med 2015; 109: 1164-1173.

15 Health and Safety Executive. Guidance Note EH76: Control of Laboratory Animal Allergy. 2015. www.hse.gov.uk/ pubns/eh76.pdf Date last accessed: February 21, 2019.

16 Nicholson PCP, Burge PS, Boyle C. Occupational Asthma: Prevention, Identification and Management: Systematic Review and Recommendations. London, British Occupational Health Research Foundation, 2010.

17 Cullinan P, Lowson D, Nieuwenhuijsen MJ, et al. Work related symptoms, sensitisation, and estimated exposure in workers not previously exposed to laboratory rats. Occup Environ Med 1994; 51: 589-592.

18 Agrup G, Belin L, Sjostedt L, et al. Allergy to laboratory animals in laboratory technicians and animal keepers. Br J Ind Med 1986; 43: 192-198.

19 Meijer E, Grobbee DE, Heederik D. Detection of workers sensitised to high molecular weight allergens: a diagnostic study in laboratory animal workers. Occup Environ Med 2002; 59: 189-195.

20 Gautrin D, Infante-Rivard C, Ghezzo H, et al. Incidence and host determinants of probable occupational asthma in apprentices exposed to laboratory animals. Am J Respir Crit Care Med 2001; 163: 899-904.

21 Newman Taylor AJ, Cullinan P, Lympany PA, et al. Interaction of HLA phenotype and exposure intensity in sensitization to complex platinum salts. Am J Respir Crit Care Med 1999; 160: 435-438.

22 Schumacher MJ, Tait BD, Holmes MC. Allergy to murine antigens in a biological research institute. J Allergy Clin Immunol 1981; 68: 310-318.

23 Larese Filon F, Drusian A, Mauro M, et al. Laboratory animal allergy reduction from 2001 to 2016: an intervention study. Respir Med 2018; 136: 71-76.

24 Feary J, Cullinan P. Laboratory animal allergy: a new world. Curr Opin Allergy Clin Immunol 2016; 16: 107-112.

25 Draper A, Newman Taylor A, Cullinan P. Estimating the incidence of occupational asthma and rhinitis from laboratory animal allergens in the UK, 1999-2000. Occup Environ Med 2003; 60: 604-605.

26 Stave GM, Darcey DJ. Prevention of laboratory animal allergy in the United States: a national survey. $J$ Occup Environ Med 2012; 54: 558-563.

27 Bonniaud P, Fabre A, Frossard N, et al. Optimising experimental research in respiratory diseases: an ERS statement. Eur Respir J 2018; 51: 1702133. 